\title{
Keine Verbesserung kognitiver Störungen
}

Fragestellung: Diese Phase-II-Studie testete die Sicherheit und Effektivität von Minocyclin bei cART-naiven Patienten mit subklinischen bis milden HIV-assoziierten kognitiven Störungen.

Hintergrund: Kognitive Störungen sind trotz der deutlichen Verbesserung der Wirksamkeit der antiretroviralen Kombinationstherapie (cART) nach wie vor bei 40-50\% der HIV infizierten Patienten zu finden [1]. Sie stellen damit unter den neurologischen Komplikationen der HIV-Erkrankung die häufigste Manifestation dar, die zu einer signifikanten Einschränkung der Lebensqualität und Berufsfähigkeit der Patienten führt. Bisherige Therapieversuche in der Behandlung von HIV-assoziierten neurokognitiven Störungen (HAND) waren erfolglos, lediglich die Initiierung einer cART führt zu einer initialen Verbesserung der Symptome. Minocyclin bietet sich als weitere Sub$\operatorname{stanz}$ an, die sowohl einen antiinflammatorischen als auch einen neuroprotektiven Effekt zumindest im Tiermodell aufweist. Darüber hinaus gibt es Hinweise auf einen direkt hemmenden Effekt auf die HIV-Replikation.

Nakasujja N, Miyahara S, Evans $S$ et al. Randomized trial of minocycline in the treatment HIVassociated cognitive impairment Neurology 2013; 80: 196-202
Patienten und Methodik: Es wurden insgesamt 73 Patienten mit subklinischer bis milder HIV-assoziierter kognitiver Störung (MSK Stadium $0,5-1$ ) in diese doppelblinde randomisierte Studie einge- schlossen. Die Patienten waren dabei noch ohne cART und wiesen CD4-Zellzahlen zwischen 250 - 500/ $\mu$ l auf. $90 \%$ der Patienten waren Frauen. Die Patienten erhielten entweder Placebo $(\mathrm{n}=37)$ oder Minocyclin $2 \times 100 \mathrm{mg} / \mathrm{d}(\mathrm{n}=38)$ über 24 Wochen. Nach zwölf und 24 Wochen erfolgten die Kontrolluntersuchungen. Der primäre Endpunkt war die Änderung im „Uganda Neuropsychological Test Battery Summary Measure" (U NP Sum). Diese Testbatterie war dabei definiert als der mittlere ZScore für folgende neuropsychologische Untersuchungen: Grooved pegboard Test, Color Trails 1 und 2, WHO-UCLA Verbal Learning Test, Zahlenmerkspanne vorwärts und rückwärts und dem Digit Symbol Test (DST). Zudem wurden mehrere Safety-Parameter und die Nebenwirkungen erfasst.

Ergebnisse: Hinsichtlich der Effektivität bestand nach 24 Wochen kein signifikanter Unterschied zwischen der Placebo- und der Verumgruppe (U NP Sum nach 24 Wochen: -0,03; $p=0,37$ ). Auch die Analyse der einzelnen neuropsychologischen Tests ergab keinen positiven Einfluss von Minocyclin auf kognitive Störungen. Minocyclin war jedoch bis auf eine Hyperpigmentierung bei 19,4\% der behandelten Patienten von keinen wesentlichen $\mathrm{Ne}$ benwirkungen begleitet und hatte keinen signifikanten Einfluss auf die CD4-Zellzahl, die Viruslast oder andere Laborparameter.

Schlussfolgerungen: Nach dieser doppelblinden randomisierten Studie ist Minocyclin zwar gut verträglich, aber nicht effektiv in der Therapie HIV-assoziierter neurokognitiver Störungen.

\section{-Kommentar von Matthias Maschke, Trier}

\section{Minocyclin ist bei dieser Fragestellung aus dem Rennen}

Die Studie ist konsistent mit einer weiteren Phase-II-Studie, die bereits 2011 publiziert wurde und ebenfalls keinen Effekt von Minocyclin 2 x 100 mg/d bei HIV-assoziierten kognitiven Störungen nachweisen konnte [3]. Somit wird es wahrscheinlich zu keiner weiteren Studie mit Minocyclin in dieser Fragestellung kommen, auch wenn eine Studie mit einer Zeitdauer von 24 Wochen möglicherweise zu kurz sein könnte, um einen neuroprotektiven Effekt sicher ausschließen zu können. Zudem wäre möglicherweise eine Kombinationstherapie mit anderen Präparaten wie zum Beispiel Deprenyl sinnvoll, da die Pathogenese der HAND sehr komplex ist und von daher nicht zu erwarten ist, dass eine einzige Substanz den Verlauf vehement ändern wird. Das Problem von Kombinationsstudien ist dabei jedoch, dass eine viel höhere Patientenzahl eingeschlossen werden müsste, um Effekte beurteilen zu können und eine Finanzierung gerade für bereits in anderer Indikation zugelassene Medikamente schwierig zu erreichen sein wird. Somit verbleibt als alleinige derzeitige Option in der Therapie von HIV Patienten mit progredienten kognitiven Defiziten die Optimierung der antiretroviralen Therapie unter Beachtung der Viruslast im Liquor und der Penetration der antiretroviralen Medi- kamente durch die Blut-Hirn-Schranke, die sich nach dem CPE (CNS pentration index, auch Letendre Index) gut beurteilen lässt [4].
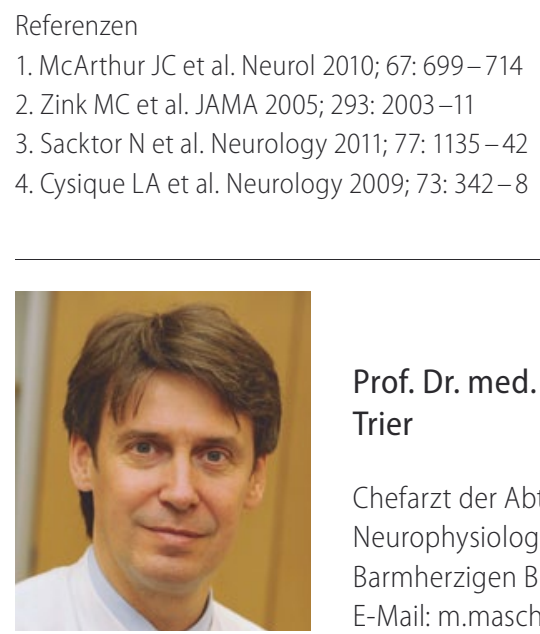

Prof. Dr. med. Matthias Maschke, Trier

Chefarzt der Abteilung für Neurologie und Neurophysiologie, Krankenhaus der Barmherzigen Brüder Trier E-Mail:m.maschke@bk-trier.de 